\title{
Measuring the Performance of Handover Mechanisms in UMTS for Diverse Traffic Services Classes to Improve QoS
}

\author{
Sher Ali \\ City University of science and \\ Information Technology, \\ Peshawar, KPK, Pakistan
}

\author{
Nasir Saleem \\ Institute of Engineering and \\ Technology, Gomal University, \\ D.I.Khan, KPK, Pakistan
}

\author{
Tayyaba Tareen \\ CECOS University, Peshawar, \\ KPK, Pakistan
}

\begin{abstract}
Cellular technologies are continuously growing. Because of these reason, 3G cellular technology has been implemented. In UMTS, services are categorized into four UMTS quality of services classes. The handover is significant characteristic of UMTS technology and is the major problem faced in implementation phase. The efficient handover process defines QoS of network. This paper addresses the analysis of different handover scenarios executed in 3G UMTS network for four different qualities of service classes. Different scenarios are created for soft and hard handovers in OPNET 14.5 Modeler simulator and conclusion are compiled for each quality of service class from analysis of results.
\end{abstract}

\section{Keywords}

UMTS, QoS, Handover, GSM, CDMA, WCDMA, RTP, FTP

\section{INTRODUCTION}

Universal Mobile Telecommunication System (UMTS) is 3G [1] mobile cellular network which is established on the basis of GSM standard and is the part of ITU's IMT-2000 family. UMTS utilizes WCDMA air interface in order to provide elevated spectral effectiveness, efficient utilization of resources and bandwidth consumption for different mobile operators. Because of this reason WCDMA [4] and UMTS [3] are used interchangeably. It supports both Time division Duplex and Frequency division for performing different operations. It is based on the IP technology having user achievable peak data rate of $350 \mathrm{kbps}$. UMTS is functional on various spectrum bands offered by the operators in flexible manner. The worldwide available bands are: $450 \mathrm{MHz}, 700$ $\mathrm{MHz}, 850 \mathrm{MHz}, 900 \mathrm{MHz}, 1700 \mathrm{MHz}, 1800 \mathrm{MHz}, 1900$ $\mathrm{MHz}, 2100 \mathrm{MHz}$ and $2600 \mathrm{MHz}$.But as the signals propagate at low frequencies, thus UMTS at $850 \mathrm{MHz}$ and $900 \mathrm{MHz}$ are more efficient bands for the low dense populated areas. Different operators can use the same common core network that provides support to multiple radio access networks together with GSM [5], EDGE, WCDMA, HSPA [6] and evaluations of these technologies. Thus provides more flexibility in diverse services. Figure 1 presents this whole concept.

\subsection{GPP AND UMTS STANDARDIZATION}

To develop a mobile technology standard this can be used.

Globally, many standardization organizations agreed together to establish an acceptable global standard. This standard is called as $3^{\text {rd }}$ generation partnership program or 3GPP [7]. The 3GPP organizations developed UMTS standard. The 3GPP air interface is responsible for Iub, Tur and Iu interfaces, UTRAN maintenance and operations requirements and BTS radio performance specifications. While the $3 \mathrm{GPP}$ core network has the responsibilities: Mobility management, call connection control signaling among mobile nodes and core network as well as among nodes, Packet related issues and definition of internetworking functions among core network and other external networks. Similarly the 3GPP terminal has to full fill the responsibilities: service capability protocol, end to end networking and USIM to mobile terminal air interface.

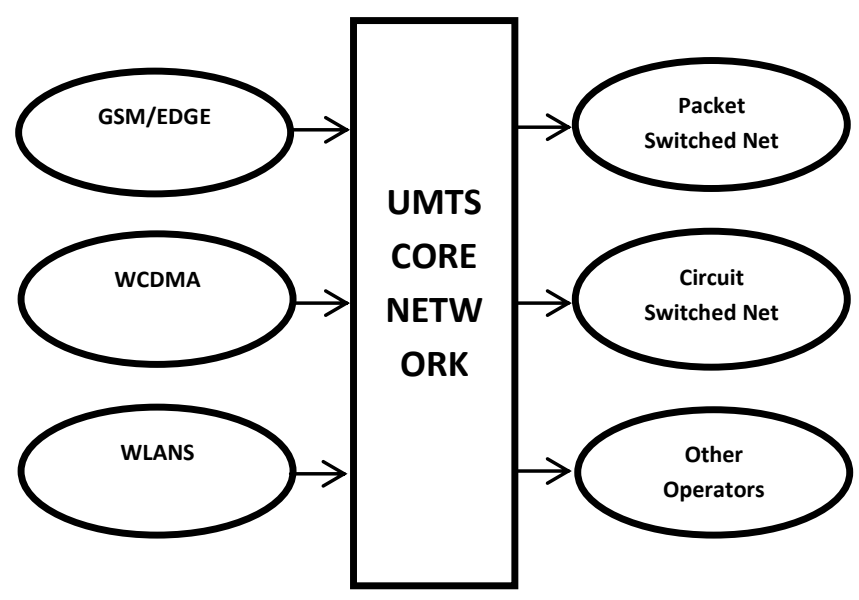

Fig.1: UMTS Multi Radio Network

\section{UMTS RELEASES}

As the Mobile technologies start having low bit rate and lower degree services because high bit rate was not required at the early stage of implementation but due to gradual increase in demands for higher bit rate and more services, the upgradation and enhancement was brought in the technologies to meet the demands. The enhanced version of technologies were given the name of next generation like $2 \mathrm{G}$ [8], similarly in the early days of after introducing the 3G [8], due to relatively lower demands for bit rate, the standard of UMTS which can fulfill the needs of that time was implemented but with gradual growth in needs for higher bit rates and other services some advancement was brought in existing release to meet the demands and given the name of next release.

\subsection{UMTS Release 99 (R99)}

The first version of UMTS was implemented in 1999 and it remains active up to early 2001 and was given name $1^{\text {st }}$ phase. This first version was called as Release 99 (R99. The main featured which made R99 dominant in $1^{\text {st }}$ phase are highlighted below: 
1. Provided better services and security.

2. Provided higher data rates

3. It contains frozen network.

\section{$2.2 \quad$ UMTS Release 2000 (R4/R5)}

With the growth of networks and subscribers, the R99 became insufficient to full fill the requirements therefore R99 is upgraded and in 2002 the complete transition was made towards R2000 (R4/R5 and given name $2^{\text {nd }}$ phase. The $2^{\text {nd }}$ phase has following features..

1. More efficient in using WCDMA resources

2. Data rate has been enhanced

3. It supports inter-release roaming

4. Speech Transcending Mechanism has been adopted that was not present in previous version

\section{UMTS HANDOVER ISSUES AND OPTIMIZATION}

The UMTS technology faces the problem of handover including both soft and hard handovers. The efficient handover mechanism determines the quality of service of any network because during active call a node can move to other cell so there must be seamless active connection among different nodes. The handover decision can be made of following reasons:

1. Decreases in signal strength

2. When mobile is moving with unpredictable fast speed.

3. When there is congestion in central active cell

4. When interference level continuously increasing In UMTS handover optimization is mechanism that obtain handover threshold. The capacity gain of system is directly related to this threshold as the threshold level and micro diversity has close relation with network performance. New optimization mechanisms can improve the QoS of network.

\section{METHODOLOGY}

The UMTS architecture is employed in OPNET (14.5) simulator by using different nodes from object palette shown in Figure 2 and 3 respectively. IP cloud was used among servers and core network to show network involvement. IP links connect IP cloud to both servers and core network (GGSN) node. These nodes were placed in such a fashion that architecture can be clearly understood. All these nodes were connected using different connection links from the object Palette. After architecture implementation, required attributes are set for each node. Applications are defined in the application definition node and were assigned to respective QoS of UMTS. The profiles were created in the profile configuration node to be used by the users. Trajectory for user equipment was defined that is based on simulation time. In OPNET modeler, a trajectory is either segment or vector based. Here the trajectory is segment-based. In order to analyze different results, node statistics were selected. A single scenario is completed in all aspects after that duplicated and attributes were set for both scenarios. The two scenarios include hard and soft handover. The simulations are compiled for different seed values and results are examined. A number of different statistics are simulated for both soft and hard handover and resulting graphs of this simulation are analyzed on the basis of QoS classes that which handover provides better services for these QoS classes. The results concluded are much helpful for the network operators to provide better QoS to the end users.

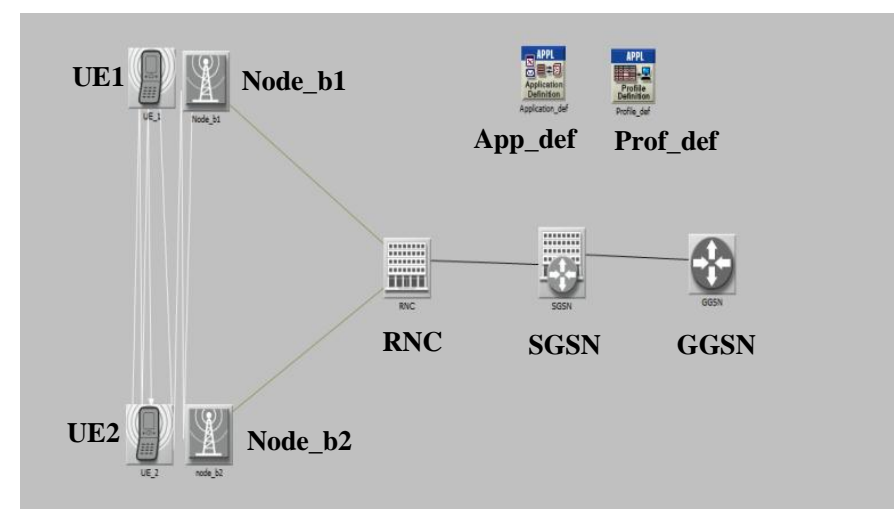

Fig 2: Scenario for conversational and streaming classes

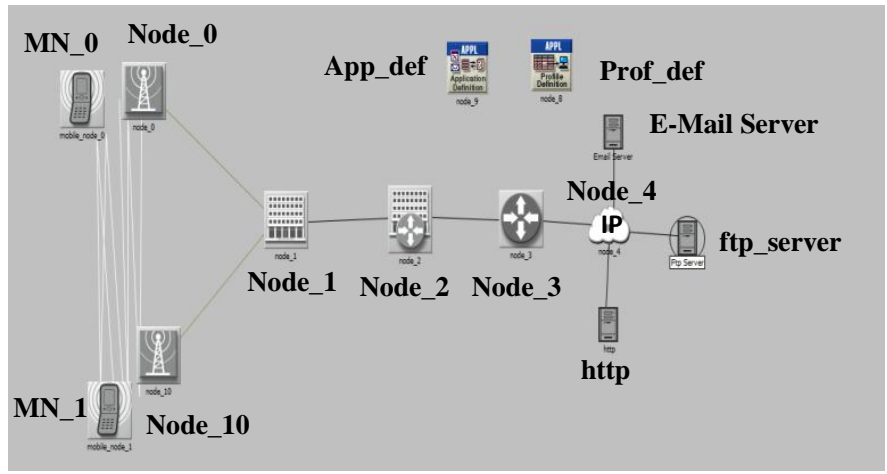

Fig. 3: Scenario for interactive and background classes

\section{HANDOVER INVESTIGATION}

In order to investigate that either handover has performed or not, active cell statics are analyzed. Active cell count shows the number of active BTS in network from which UE is receiving data simultaneously. These active BTS are called active set of UE and is represented in figure 4. We can see in network scenario that UE initially connected to only BTS at beginning of simulations. Therefore value of active set of the UE is 1 for both handovers as shown in upper and lower part of the figure 4; the upper portion represents hard handover and lower part shows soft handover. As we know that in hard handover the UE is attach to only one BTS at time so initially count of active set is 1 . When the signal level become lowered than threshold level for a UE in existing active cell it just terminates connection with that BTS and establishes a new connection with another BTS from which it can get enough signals strength. At this time again count of the active set is 1 because only one BTS is there from which it is receiving signals. During handover, the connection is BREAK BEFOR MAKE so this analysis shows that count of active set of UE throughout hard handover simulation will always be 1 can be viewed in figure 4 . In case of soft handover initially UE is attached to only one cell therefore value in active set of the UE is 1 . But as UE starts moving, it starts receiving signals from more than one cell, at that time count of active set is updated to 2 as UE is receiving signals from 2 cells simultaneously. When the signal strength for UE begins to decrease in one cell and increase from other cell to a specific threshold level, UE also makes connection with that cell and breaks connection with previous cell. After this handover count of active set of UE is again 1 because again UE is 
attached to only one cell. The soft handover follows the rule of MAKE BEFOR BREAK so count of active set varies throughout simulations as shown in lower part of figure 4 .

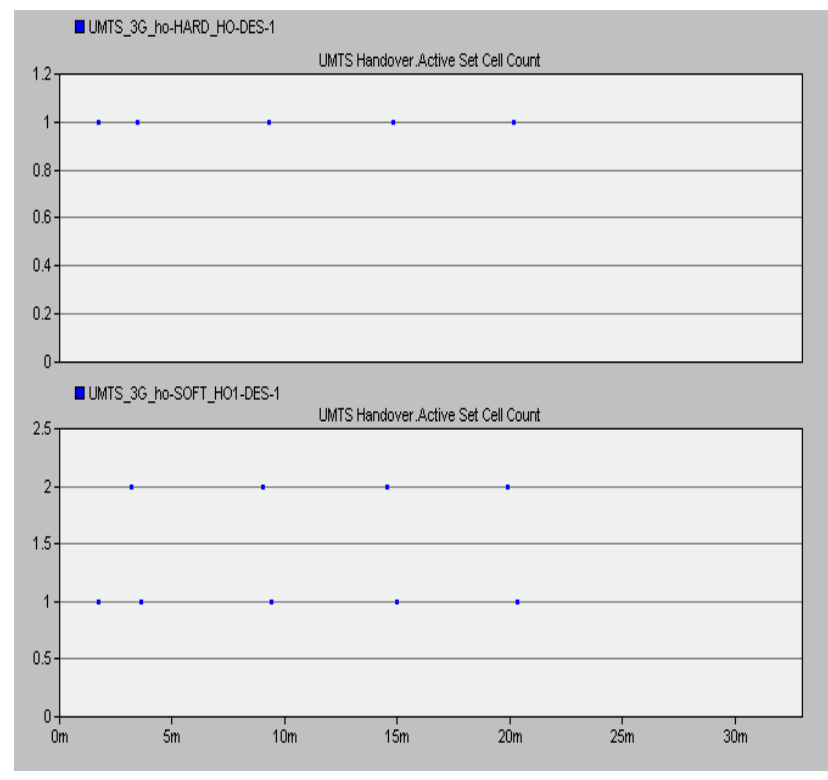

Fig 4: Active Set Cell Count

\section{PERFORMANCE ANALYSIS OF OPNET SIMULATIONS}

Soft and hard handovers conditions are simulated in OPNET and after simulated statistics analysis conclusions are made on the basis of QoS that which handover provides better services. The end results obtained from these analyses will be very advantageous for end user in terms of achieving better QoS. Two scenarios are built in simulator Scenario 1:

- QoS

0 (Conversational Class)

- QoS

1 (Streaming Class)

Scenario 2

- QoS 2 (Interactive class)

- QoS 3 (Background class)

We have used voice from conversation class, video conferencing from streaming class, email and http from interactive class and ftp from background class.

\subsection{ANALYSIS OF QOS 0 (CONVERSATIONAL CLASS)}

We run the scenario of voice applications selecting different seed value for each simulation run. Every time we changed seed value, we found different results graph for same individual statistics and same scenario then graph with best results is selected for analysis. The QoS 0 is for voice; therefore, before making any conclusion, some factors are under consideration which includes packet end-to-end delay, traffic sent and traffic received. The total voice packet network delay is time at which sender node provides packet to RTP to time receiver receives it from RTP is packet end-toend delay. As each QoS class has different levels of losses and delay sensitivity therefore packet end-to-end delay is analyzed separately for each QoS application. From the figure 5 it is clear that soft handover gives better results than hard handover showing lesser packet end-to-end delay as compared to hard handover.

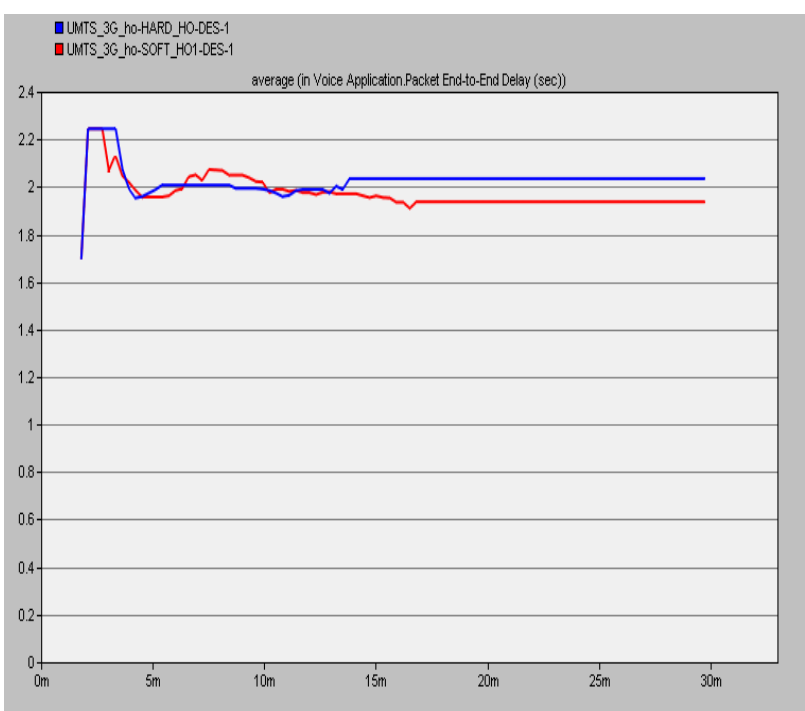

Fig. 5: Packet End-to-End Delay in Sec

Average number of packets per second submitted to transport layer by voice application of node is said to be traffic sent measured in packets/sec, this statistics basically tells us that how much a node is capable of sending data in form of packets during handover. It is clear from figure 6 that for almost throughout simulation time node is sending equal voice packets in both soft and hard handovers.Similarly Average number of packets/second forwarded to voice application by transport layer in this node is known as traffic received. It is shown in the figure 7 that for almost all simulations the soft handover provides better results in terms traffic received.

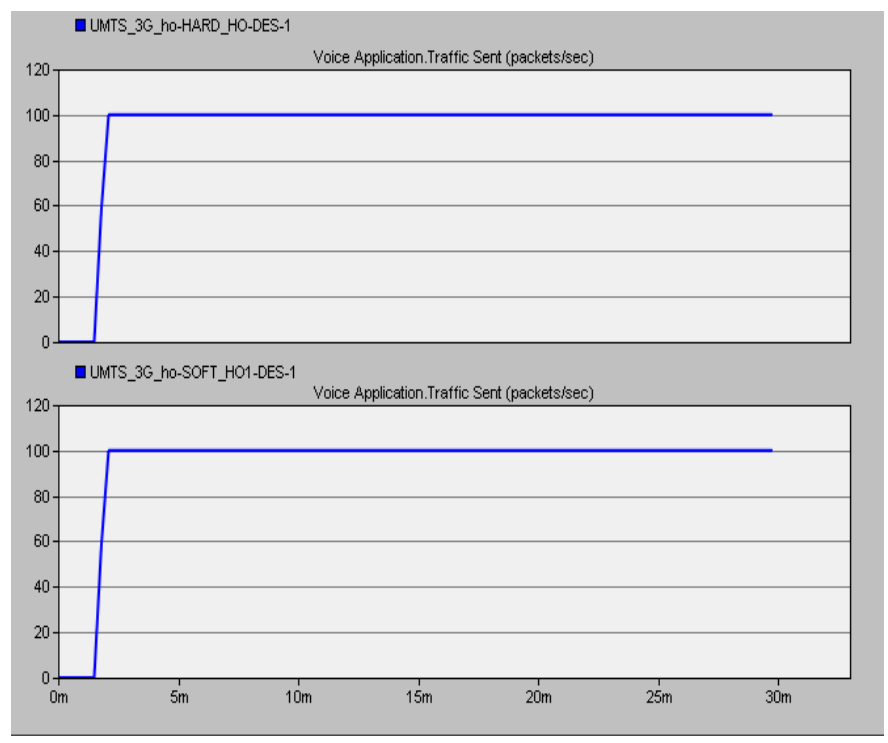

Fig 6 Traffic Sent (Packets/Second) 


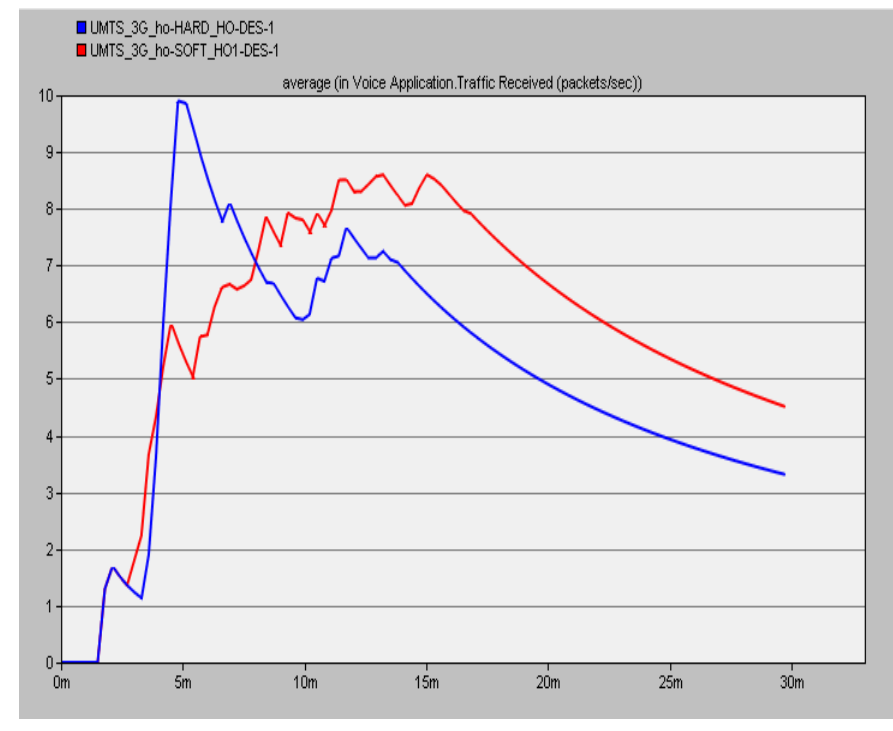

Fig 7 Traffic Received (Packets/Second)

\subsubsection{Conclusions for QoS 0}

As conversational class which includes real time services such as voice application, should not have delay parameter. So from above results it is concluded that soft handover which has less delay than hard handover, is suitable for QoS 0 conversational class. The traffic received graph shows that more number of packets is being received in soft handover due to less delay. Hence soft handover is better for QoS 0 .

\subsection{ANALYSIS OF QOS 1 \\ (STREAMING CLASS)}

The QoS 1 is associated with the streaming video; therefore, before making any conclusions following statics must be analyzed. Packet end-to-end delay, packet sent and received. From figure 8 it is clear that soft handover gives better results than hard handover showing lesser packet end-to-end delay as compared to hard handover.

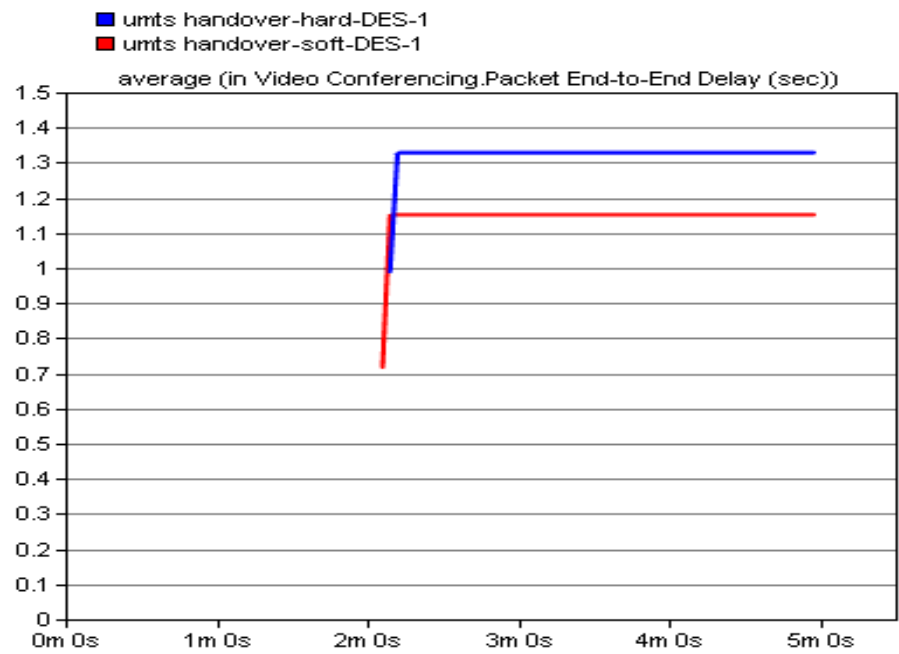

Fig 8 Video Packet End-to-End Delay in seconds

Figure 9 shows that almost throughout simulations time node is sending equal video packets in soft and hard handover. While Figure 10 shows that UE receives higher number of packets during hard handover as compared to soft handover but with a bit of delay in it.

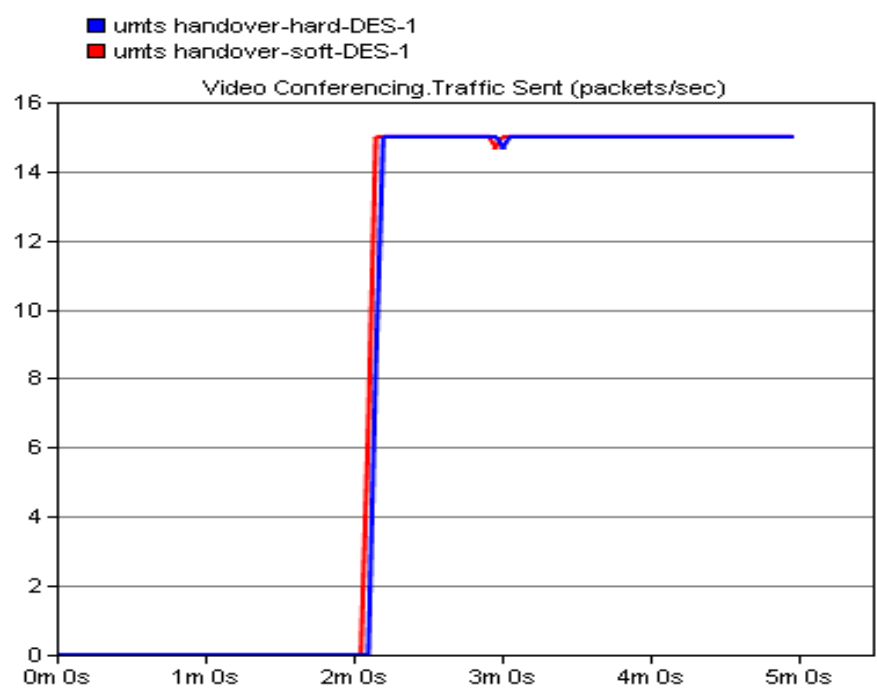

Fig 9 Video Traffic Sent (Packets/second)

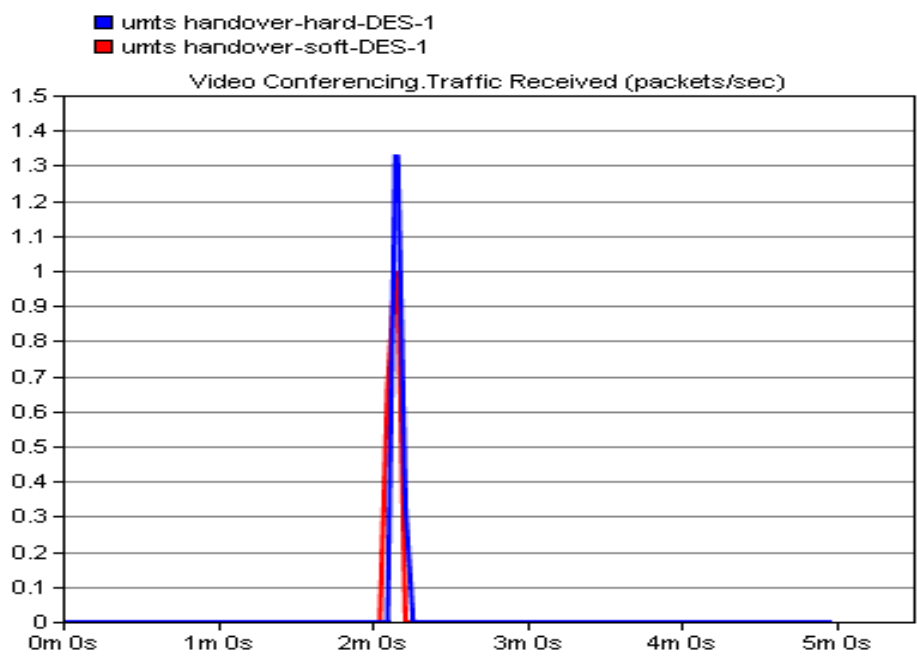

Fig 10 Video Traffic Received (Packets/Seconds)

\subsubsection{Conclusions for QoS 1}

As the streaming class which includes real time streaming services such as video streaming, buffering etc. can afford a bit of delay, so from above results it is concluded that the hard handover which has more delay than soft handover, is suitable for QoS 1 streaming class because it receives more data with a bit of delay which is affordable in streaming class. Hence hard handover is better choice for QoS 1 video streaming.

\subsection{ANALYSIS OF QOS 2 (INTERACTIVE CLASS)}

The QoS 2 belongs to interactive e-mail class and there are two types of e-mail services including interactive e-mail (e.g. Chat) and background e-mail (Downloading e-mails). Thus before making any conclusions following analysis of statistics must be considered. The figure 11 shows that for almost all the simulations the hard handover gives better results in terms traffic sent, it means more packets are sent at the sending UE during hard handover as compared to soft handover. More traffic is sent during hard handover as compared to soft handover because this QOS class is very less sensitive to delay therefore it does not matter whether the connection break before make or make before break. 


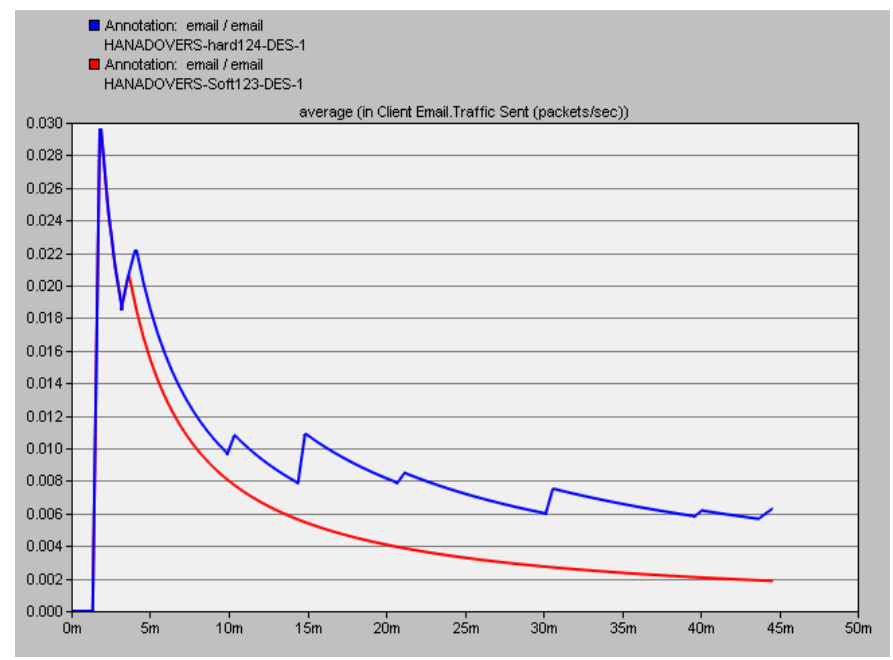

Fig 11 Traffic Sent (Packets/Seconds)

It is shown in figure 12 that for almost all the simulations the hard handover gives better results in terms traffic received; it means more packets are received at the receiving UE during hard handover as compared to soft handover.

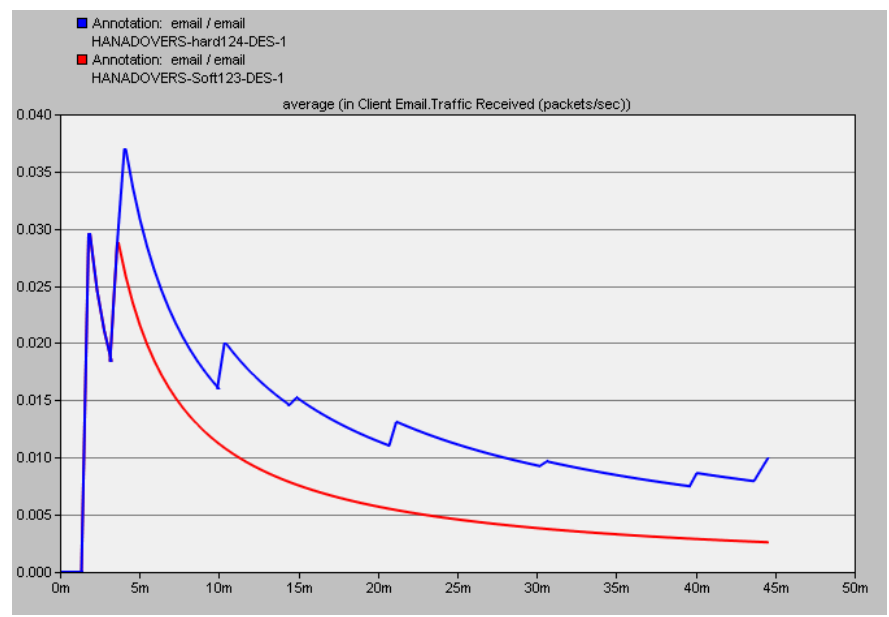

Fig 12 Traffic Received (Packets/Second)

\subsubsection{Conclusions for $\mathrm{QoS} 2$}

In interactive class that includes web browsing services such as http, email etc. the loss of information or packets must be reduced because damaging or loss of information degrades quality of service. So from results it is concluded that hard handover which has less delay and less uploading and downloading response time for more data transfer, than in soft handover, is suitable for QoS 2 the interactive class. The traffic sent and received graph shows that more number of packets are being received and sent in hard handover with less delay. Hence hard handover is better for QoS 2 or the email application.

\subsection{ANALYSIS OF QOS 3 (BACKGROUND CLASS)}

The QoS 3 is based on applications associated with ftp so following conclusions are made on basis of analyzed data. It can be conclude from figure 13 that for almost throughout the simulations time the node is sending more FTP packets in soft handover as compared to hard handover .It is shown in one of the graph chosen from different runs at different seeds.

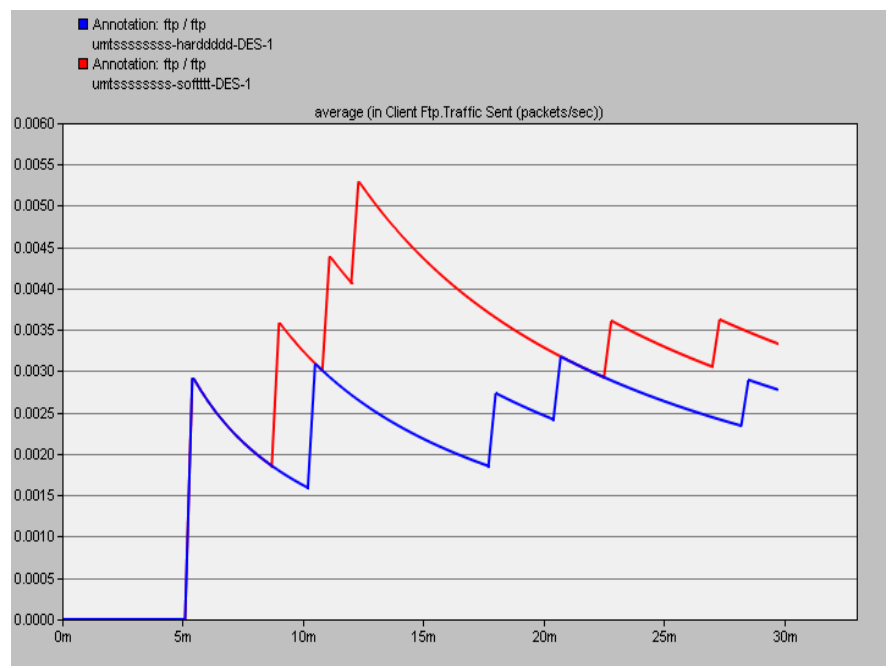

Traffic Received (packets /sec) is the statistic which is define as the Average number of packets per second forwarded to FTP applications by the transport layers in the network is known as Traffic Received (packets /sec). The figure 14 clearly shows that the UE receives higher number of packets during hard handover.

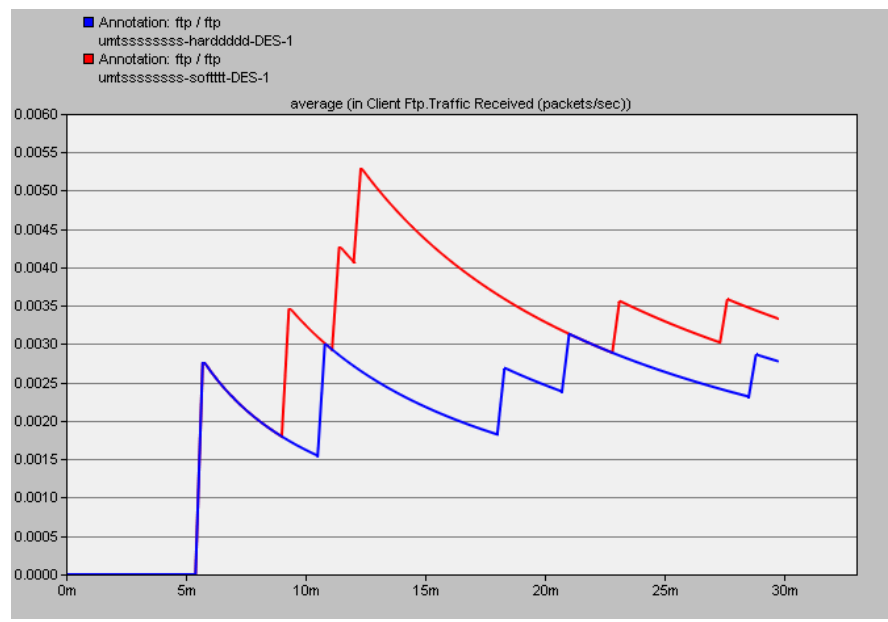

Fig 14 Traffic Received (Packets/Second)

\subsubsection{Conclusions for QoS 3}

The Background class includes file downloading and uploading services such as ftp application, thus loss the information or packets must be reduced to obtain better QoS. From above results it is concluded that soft handover has less delay and less response time for uploading and downloading is suitable for QoS 3 background class.

\section{CONCLUSION AND FUTURE WORK}

OPNET modeler 14.5 is used as a simulation tool and two scenarios are built, one for UMTS, QoS 0 class and QoS 1 class while the 2nd scenario is built for QoS 2 class and QoS 3 class. Both soft and hard handover scenarios are built for each quality of services class. Using network entities dragging from object share palate in to the network area. After the scenarios are completed and individual statistics are choose to analyze results for many parameters. Handovers are 
performed for each class .Only one data type is taken for analysis from each QoS class e.g. voice, video, email and ftp applications are taken from QoS 0, 1, 2, 3 classes respectively . Every QoS class is analyzed in terms of many parameters like traffic sent and received, end-to-end delay variation, upload response time and download response time. The soft handover best suited for conversation (Voice) QoS 0 class applications and background QoS 3 class. The hard handover is best suited for QoS1 and QoS2. In future, problems related to capacity of users and coverage area of this network will be brought under research and solution for these problems will be proposed. Furthermore the analysis and comparison of sector to sector and cell to cell handover will be done for all the four classes in terms of transmission power, delays and total throughputs. 3-sectors and 6-sectors cells will be used for inter sector handovers and thief effects will be analyzed in both the 3-sectors and 6-sectors cells. A more efficient adoptive handover algorithm can be suggested which can adopt itself according to data type used.

\section{REFERENCES}

[1] Rapeli, "UMTS- a path to $3^{\text {rd }}$ generation mobile communication system of the $21^{\text {st }}$ century", 2006, PWC 2: p.34-42.
[2] Shin-mingCheng,"Key management for UMTS MBMS". 2008, Wireless communication 33: 75-77.

[3] Harmatos, J. Anal, 2002. "Planning of UMTS core networks The 13th IEEE International Symposium on Personal, Indoor and Mobile Radio Communications. PIMRC 39.

[4] Binucci and Hiltunen, "Soft handover gain in WCDMA". 2003, vehicular technology 33: 65-68.

[5] GuifenGu, GuiliPeng, "The survey of GSM wireless communication system" Computer and Information Application (ICCIA), 2010 International Conference on3-5 Dec. 2010

[6] Shah, S.I. "UMTS: High Speed Packet Access (HSPA) Technology" IEEE, 2008

[7] Ludwig, R.; Ekstrom, H.; Willars, P.; Lundin, N.” An Evolved 3GPP QoS Concept"IEEE Conference, 2006, pg 388-392.

[8] Kalavakunta, R.; Kripalani, A. "Evolution of mobile broadband access technologies and services considerations and solutions for smooth migration from 2G to 3G networks", 2005, IEEE Conference, pg 144 149. 